\title{
Elastic Properties of Nanowires
}

\author{
Alexandre F. da Fonseca* and C. P. Maltat \\ Instituto de Física, Universidade de São Paulo, \\ USP, Rua do Matão Travessa R 18\%, \\ CEP 05508-900, São Paulo, SP, Brazil \\ Douglas S. Galvãd \\ Instituto de Física 'Gleb Wataghin', \\ Universidade Estadual de Campinas, \\ UNICAMP CEP 13083-970, Campinas, SP, Brazil
}

\begin{abstract}
We present a model to study Young's modulus and Poisson's ratio of the composite material of amorphous nanowires. It is an extension of the model derived by two of us [Da Fonseca and Galvão, Phys. Rev. Lett. 92, 175502 (2004)] to study the elastic properties of amorphous nanosprings. The model is based on twisting and tensioning a straight nanowire and we propose an experimental setup to obtain the elastic parameters of the nanowire. We used the Kirchhoff rod model to obtain the expressions for the elastic constants of the nanowire.
\end{abstract}

PACS numbers: $62.25 .+\mathrm{g}, 61.46 .+\mathrm{w}, 46.70 . \mathrm{Hg}$ 


\section{INTRODUCTION}

The growing interest in the field of nanoscience comes, in part, from the discovery of onedimensional carbon nanotubes $\frac{1}{\underline{\underline{1}}}$ and its special physical properties as, for example, the exceptionally high Young's modulus $(\sim 1 \mathrm{TPa})$. It has attracted the attention of the scientific community to the wide potential of the nanostructures to diverse scientific and technological applications. $2 \cdot 3 \cdot 4 \cdot 5 \cdot 6.7$

Among the different types of nanostructures, carbon nanotubes, nanowires and nanosprings present special mechanical properties. In order to understand their macroscopic mechanical behavior it is necessary to study their elastic properties at nanoscale. Scanning transmission microscopy (STM), atomic force microscopy (AFM) and transmission electron microscopy (TEM) have been used to manipulate and characterize the properties of indi-

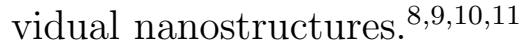

In this paper, we present a model for the study of the elastic properties of amorphous straight nanowires. We propose an experimental setup that can be used to measure the Young's modulus and the Poisson's ratio of the composite material of an amorphous nanowire in two situations.

Our proposal is based on two situations involving a twisted nanowire. The first situation is a static configuration in which the nanowire is maintained twisted by a given torque. From the equations of equilibrium for a filament in this situation, we obtain an expression for the elastic parameters of the nanowire. The second situation involves the twisting and tensioning of a nanowire. In order to obtain a second expression for the elastic parameters of the nanowire, we use the well known fact that a twisted rod becomes unstable if its twist density reaches a given critical value.

The Kirchhoff rod mode ${ }^{12}$ is used to derive simple equations for the elastic constants of the composite material of an amorphous nanowire in the two above mentioned configurations. These equations involve the geometry of the twisted nanowire and the forces and torques that hold the nanowire deformed. Since we are considering amorphous nanowires, a continuous mechanical model is appropriate to study the elastic properties of these systems. The present work is a direct extension of a previous work where the Kirchhoff rod model was used to 
derive two expressions for measuring the Young's modulus and Poisson's ratio of amorphous nanosprings. 13

\section{THE MECHANICAL ROD MODEL}

The Kirchhoff model is a theory for small deformations of inextensible thin elastic rods. It has been extensively used to study the statics and the dynamics of different kinds of continuous filaments with applications ranging from Biology 14.15.16.17.18.19.20 to Engineering. ${ }^{21.22}$

The Kirchhoff equations are derived from the application of Newton's laws of mechanics to a thin elastic rod in the approximation of small curvature. ${ }^{23}$ An additional condition is needed to solve the set of differential equations and it comes from the constitutive relation of linear elasticity theory that relates the moments to strains. ${ }^{23}$ These equations contain the forces and torques, plus a triad of vectors describing the deformations of the rod. For a circular homogeneous rod, they are given by

$$
\begin{aligned}
& \mathbf{F}^{\prime \prime}=\rho A \ddot{\mathbf{d}}_{3} \\
& \mathbf{M}^{\prime}+\mathbf{d}_{3} \times \mathbf{F}=\rho I\left(\mathbf{d}_{1} \times \ddot{\mathbf{d}}_{1}+\mathbf{d}_{2} \times \ddot{\mathbf{d}}_{2}\right) \\
& \mathbf{M}=E I k_{1} \mathbf{d}_{1}+E I k_{2} \mathbf{d}_{2}+\frac{E I k_{3}}{1+\sigma} \mathbf{d}_{3},
\end{aligned}
$$

where $\mathbf{F}$ and $\mathbf{M}$ are the total force and torque across the cross-sections of the rod, respectively. $\mathbf{d}_{i}$ with $i=1,2,3$ form a triad of unitary vectors defined in each cross-section where $\mathbf{d}_{3}$ is the vector tangent to the axis of the rod and $\mathbf{d}_{1}$ and $\mathbf{d}_{2}$ lie on the plane of the crosssection along, for example, its principal axes. $E$ and $\sigma$ are the Young's modulus and the Poisson's ratio of the composite material of the rod. $\rho$ is the mass density of the rod, and $I$

is the moment of inertia of the cross section, here considered as being circular of area $A$. If $r$ is the radius of the cross section,

$$
I=\frac{\pi r^{4}}{4}
$$

In Eqs. (10), the prime and the dots denote the derivative with respect to the arc-length $s$ of the rod and to the time $t$, respectively. $k_{i}, i=1,2,3$, are the components of the socalled twist vector, $\mathbf{k}$, which defines the variation of the director basis $\left\{\mathbf{d}_{1}, \mathbf{d}_{2}, \mathbf{d}_{3}\right\}$ with the arc-length $s$ through the expression: $\mathbf{d}_{i}^{\prime}=\mathbf{k} \times \mathbf{d}_{i}, i=1,2,3$. The Eq. (1c) is valid for an 
intrinsically straight rod, i. e., a rod that is straight when free from the action of stresses. That is the case of an amorphous straight nanowire.

\section{THE EXPERIMENTAL MODEL}

One of the advantages of our proposal is the fact that with an unique experimental setup we can perform two measurements that, combined, can give the elastic parameters of the composite material of the nanowire.

Fig. 11 displays the proposed scheme to measure the Young's modulus and the Poisson's ratio of the nanowire. Two experiments can be performed with the same experimental setup.

The first experiment is based on a static situation in which the nanowire is twisted a given number $n$ of turns through the application of a torque $\mathbf{M}$ along the direction of the axis of the nanowire. The second experiment is based on the application and control of a force of tension $\mathbf{T}$ on a twisted nanowire.

In order to derive the expression to be used in the first experiment, we have to obtain an equilibrium solution of the Kirchhoff equations, $i$. e., a solution for the case where the time derivatives are eliminated from the Eqs. (11). The equilibrium solution corresponding to a twisted straight rod is given by

$$
\begin{aligned}
& \mathbf{k}=\gamma \mathbf{d}_{3}, \\
& \mathbf{F}=T \mathbf{d}_{3}, \\
& \mathbf{M}=(1+\sigma)^{-1} E I \gamma \mathbf{d}_{3},
\end{aligned}
$$

where $\gamma$ is the twist density of the rod that is defined by:

$$
\gamma=\frac{n}{L}
$$

where $n$ is the number of turns produced by the application of the torque $\mathbf{M}$, and $L$ is the total length of the nanowire. $T$ is a force of tension or compression applied along the axis of the rod and it is a free parameter in the static situation. The value of $T$ will be important in the second experiment which involves the condition for the stability of the equilibrium solution given by Eqs. (3). 
We rewrite the Eq. (3c) in order to obtain an expression for the elastic constants in terms of the applied torque and the twist density:

$$
\frac{E}{\sigma+1}=\frac{4\|\mathbf{M}\|}{\pi r^{4} \gamma}
$$

where we have used the Eq. (2) to substitute the expression for the moment of inertia, $I$, in terms of the cross section radius, $r$.

In the first experiment, we apply a given torque $\mathbf{M}$ to twist a nanowire a given number $n$ of turns. By measuring the value of $\|\mathbf{M}\|$ and calculating the resultant twist density $\gamma$ in a nanowire of cross-section radius $r$, we obtain the ratio $E /(\sigma+1)$ of the nanowire using Eq. (515). The next experiment will provide the other relation between $\sigma$ and $E$.

In Eq. (44), $n$ does not have to be an integer. Any fraction (for instance, a torsion of about 30 degrees) of a turn can be considered in the experiment because the twist density is a real number. Our proposal is based only on elastic deformations of the nanowire in the linear regime. Therefore, the plastic regime can be avoided by making a small number of turns or a fraction of a turn on the nanowire. It is important to measure the magnitude of the applied torque that produces a given twist density. It is also important to maintain one of the extremities held fixed in order to prevent the relaxation of the nanowire.

In order to discuss the second experiment, let us briefly introduce the dynamical method of stability analysis developed by Goriely and Tabor, ${ }^{24,25}$ to study the stability of equilibrium solutions of the Kirchhoff equations for twisted rods. Their method is based on an expansion of the director basis of the deformed configuration around the director basis of the stationary solution, and they use the Eqs. (1) to obtain different sets of equations for each power of a given pertubative parameter $\epsilon$. The linear analysis can be performed studying the equations at order $O(\epsilon)$. Nonlinear analysis can be performed using the equations for higher orders of $\epsilon$. From the linear and nonlinear analysis of a twisted straight rod, Goriely and Tabor ${ }^{25}$ obtained the following expression for the twist density $\gamma_{C}$ above which a finite rod becomes unstable:

$$
\gamma_{C}=(1+\sigma) \sqrt{\frac{1}{L^{2}}+\frac{4 T}{E I}} .
$$

We can see now that the value of the tension $T$ is very important for the stability of a twisted rod. High values of $T$ leads to high values for $\gamma_{C}$, so that by increasing the tension 
on a twisted rod, we can prevent it from buckling due to instability. In order to define the second experiment, we rewrite the Eq. (6) to obtain an expression for the critical value, $T_{C}$, of the force of tension, for which a twisted rod, with fixed twist density $\gamma$, becomes unstable:

$$
T_{C}=\frac{E I}{4}\left(\frac{\gamma^{2}}{(1+\sigma)^{2}}-\frac{1}{L^{2}}\right) .
$$

The second experiment is also based on twisting a nanowire but here we apply and control the magnitude of the force of tension on it. The scheme is shown in Fig. 1. In order to obtain the critical value, $T_{C}$, of the force of tension, we must perform the following steps: 1 ) we apply a force of tension $T$ along the axis of the nanowire maintaining it tensioned; 2 ) a torque is applied along the axis of the nanowire so as to rotate the nanowire a number $n$ of turns, thus producing a twist density $\gamma$, as in the previous experiment. Keeping the torque constant fixes the value of $\gamma$, and we do not need to measure the magnitude of the applied torque; 3) the magnitude of $T$ is slowly decreased until the nanowire becomes unstable and gets buckled. The value of $T$ such that the nanowire becomes unstable is the critical value $T_{C}$ that can be used in the eq. (7) to obtain another relation between $E$ and $\sigma$.

Combining the results obtained using the Eqs. (5) and (7), we obtain the values for the Young's modulus, $E$, and the Poisson's ratio, $\sigma$, of the nanowire.

\section{DISCUSSION AND CONCLUSION}

The great potential for scientific and technological applications of different types of nanostructures, has motivated a large amount of work on the physical and chemical properties of nanostructures. In future technological applications, any device composed of onedimensional nanostructures might take advantage of the individual properties, specially those related to the elastic responses to bending or twisting.

Manipulation of individual nanowires is a great challenge in characterizing these systems. STM and AFM have been the dominant approaches towards nanomanipulation. 8.10 Bending of nanowires $\frac{8,11,27,28}{2}$ and stretching of nanocoils ${ }^{29}$ have been reported in the literature but there is no report about the twisting nanowire experiments. Nakatani and Kitagawa ${ }^{30}$ recently presented an atomistic study of twisting a nanowire using molecular dynamics. 
We hope that our proposal can motivate experimentalists to develop methods for twisting individual nanowires.

An important advantage of our proposed model is the fact that with an unique experimental setup, we can make two experiments to obtain directly the Young's modulus and the Poisson's ratio of the nanowire. Since an amorphous nanowire is modeled as a continuous rod, these two elastic constants completely characterize ${ }^{26}$ the elastic behavior of the nanowire.

If we wish to obtain the shear modulus $\mu$ of the composite material of the nanowire, we can use the following relation: ${ }^{26}$

$$
\mu=\frac{E}{2(\sigma+1)} .
$$

We can see that Eq. (5) gives directly the expression for $2 \mu$, thus $\mu$ can be directly measured using the first scheme proposed.

Our model can be used to test and confirm previous measurements of the elastic parameters of an amorphous nanowire. For example, alternating eletrical fields have been used to excite mechanical resonance in $\mathrm{SiO}_{2}$ nanowires 27 in order to measure the Young's modulus of a nanowire having diameter $\sim 100 \mathrm{~nm}$ and length over $10 \mu \mathrm{m}$. They obtained a value close to $47 \mathrm{GPa}$ for the Young's modulus of these $\mathrm{SiO}_{2}$ nanowires that is smaller than that for the bulk amorphous $\mathrm{SiO}_{2}(\sim 72 \mathrm{GPa})$. The Young's modulus of thinner $\mathrm{SiO}_{2}$ (diameter $\sim$ $50 \mathrm{~nm}$ ) was measured using TEM ${ }^{8}$ and the obtained value (approximatelly $28 \mathrm{GPa}$ ) is even smaller than that for the bulk case.

In other example, a reported increase of the Young's modulus of different nanowires of diameters smaller than $70 \mathrm{~nm}$ has been explained in terms of surface tension effects $\frac{31}{\underline{\underline{w}}}$ Our proposed experiments can be useful to test these effects since they are not based on bending and, therefore, do not generate variations in the surface area of the nanowire.

Conversely, our model can be used to estimate the torque required to twisting a given nanowire if its Young's modulus and Poisson's ratio are known. This can be very useful in technological applications where individual nanowires can be subjected to forces and torques.

In summary, we have presented an experimental scheme to measure the Young's modulus and the Poisson's ratio of the material that composes an amorphous nanowire. Two schemes were proposed based on the twisting of a nanowire. One scheme is based on a static config- 
uration of a twisted nanowire and the other is based on the conditions for a twisted rod to become unstable. The Kirchhoff rod model was used to obtain simple expressions relating the Young's modulus and the Poisson's ratio of the material to the forces and torques that hold the nanowire deformed. These two elastic constants completely characterize the elastic properties of an individual amorphous nanowire. It is, therefore, an important contribution for the development of mechanical applications in nano-engineering.

\section{Acknowledgments}

This work was partially supported by the Brazilian agencies FAPESP, CNPq, IMMP/MCT, IN/MCT and FINEP. 
* Eletronic mail: afonseca@if.usp.br

$\dagger$ Eletronic mail: coraci@if.usp.br

$\ddagger$ Eletronic mail: galvao@ifi.unicamp.br

1 S. Iijima, Nature (London) 354, 56 (1991).

2 R. H. Baughman, A. A. Zakhidov, and W. A. de Heer, Science 297, 787 (2002).

3 A. S. Edelstein, and R. C. Cammarata, Nanomaterials: Synthesis, Properties and Applications (Institute of Physics Publishing, Bristol, 1996).

4 K. W. Wong, X. T. Zhou, F. C. K. Au, H. L. Lai, C. S. See, and S. T. See, Appl. Phys. Lett. 75, 2918 (1999).

5 M. S. Gudiksen, L. J. Lauhon, J. Wang, D. C. Smith, and C. M. Lieber, Nature (London) 415, 617 (2002).

6 H. I. Liu, D. K. Biegelsen, F. A. Ponce, N. M. Johnson, and R. F. W. Pease, Appl. Phys. Lett. 64, 1383 (1994).

7 L. Samuelson, Mater. Today 6 (10), 22 (2003).

8 Z. L. Wang, R. P. Gao, P. Poncharal, W. A. de Heer, Z. R. Dai, and Z. W. Pan, Mater. Sci. Eng. C 16, 3 (2001).

9 P. Poncharal, Z. L. Wang, D. Ugarte, and W. A. de Heer, Science 283, 1513 (1999).

10 X. Li, P. Nardi, C.-W. Baek, J.-M. Kim, and Y.-K. Kim, J. Micromech. Microeng. 15, 551 (2005).

11 J. Song, X. Wang, E. Riedo, and Z. L. Wang, Nano Letters 5, 1954 (2005).

12 G. Kirchhoff, J. Reine Anglew. Math. 56, 285 (1859).

13 A. F. da Fonseca, and D. S. Galvão, Phys. Rev. Lett. 92, 175502 (2004).

14 W. K. Olson, Curr. Opin. Struct. Biol. 6, 242 (1996).

15 I. Tobias, D. Swigon, and B. D. Coleman, Phys. Rev. E 61, 747 (2000).

16 A. F. Fonseca, and M. A. M. de Aguiar, Phys. Rev. E 63, 016611 (2001).

17 A. F. da Fonseca, and M. A. M. de Aguiar, Physica D 181, 53 (2003).

18 A. F. da Fonseca, C. P. Malta, and M. A. M. de Aguiar, Physica A 352, 547 (2005). 
19 A. Goriely, and M. Tabor, Phys. Rev. Lett. 80, 1564 (1998).

20 T. McMillen, and A. Goriely, J. Nonlin. Sci. 12, 241 (2002).

21 J. Coyne, IEEE J. Ocean. Eng. 15, 72 (1990).

22 Y. Sun, J. W. Leonard, Ocean. Eng. 25, 443 (1997).

23 E. H. Dill, Arch. Hist. Exact. Sci. 44, 2 (1992).

24 A. Goriely, and M. Tabor, Physica D 105, 20 (1997); Physica D 105, 45 (1997).

25 A. Goriely and M. Tabor, Nonlinear Dynamics 21, 101 (2000).

26 L. D. Landau and E. M. Lifshitz, Theory of Elasticity, Reed Educational and Professional Publishing Ltd, Oxford (1986).

27 D. A. Dikin, X. Chen, W. Ding, G. Wagner, and R. S. Ruoff, J. Appl. Phys. 93, 226 (2003).

28 X. Chen, S. Zhang, G. J. Wagner, W. Ding, and R. S. Ruoff, J. Appl. Phys. 95, 4823 (2004).

29 X. Chen, S. Zhang, D. A. Dikin, W. Ding, R. S. Ruoff, L. Pan and Y. Nakayama, Nano Letters 3, 1299 (2003).

30 A. Nakatani, and H. Kitagawa, presented at XXI International Congress of Theoretical and Applied Mechanics, Warsaw, Poland 2004 (unpublished).

31 S. Cuenot, C. Frétigny, S. Demoustier-Champagne, and B. Nysten, Phys. Rev. B 69, 165410 (2004). 
List of figure captions

FIGURE 1: Outline of the experimental setup to measure the elastic constants $E$ and $\sigma$. A torque $\mathbf{M}$ is applied along the axis of the rod, creating a twist density $\gamma$. $\mathbf{T}$ is the force of tension. 


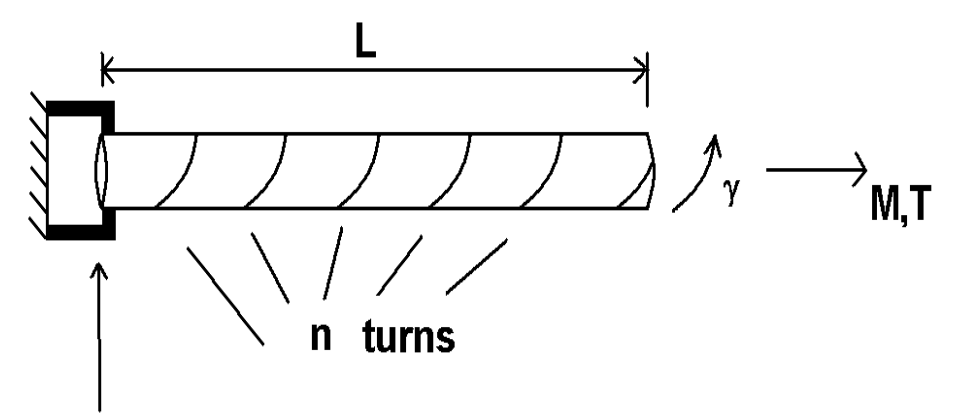

Fixed extremity

FIG. 1: Outline of the experimental setup to measure the elastic constants $E$ and $\sigma$. A torque $\mathbf{M}$ is applied along the axis of the rod, creating a twist density $\gamma . \mathbf{T}$ is the force of tension. 